\title{
Gauge and Gravitational Anomalies and Hawking Radiation of Rotating BTZ Black Holes
}

\author{
M.R. Setare * \\ Department of Science, Payame Noor University. Bijar. Iran
}

\begin{abstract}
In this paper we obtain the flux of Hawking radiation from Rotating BTZ black holes from gauge and gravitational anomalies point of view. Then we show that the gauge and gravitational anomaly in the BTZ spacetime is cancelled by the total flux of a 2-dimensional blackbody at the Hawking temperature of the spacetime.
\end{abstract}

${ }^{*}$ E-mail: rezakord@ipm.ir 


\section{Introduction}

It is commonly believed that any valid theory of quantum gravity must necessary incorporate the Bekenestein-Hawking definition of black hole entropy [1, 2] into its conceptual framework. However, the microscopic origin of this entropy remains an enigma. Many efforts have been devoted to this theme. An approach to the Hawking radiation is to calculate the energy-momentum tensor in the black hole backgrounds. However the usual expression for the stress tensor includes singular products of the field operators for stress tensor. Renormalization theory of the stress tensor claims to solve this problem, but it must be mentioned that the usual scheme of renormalization includes complexity and somewhat ambiguity. In semiclassical framework for yielding a sensible theory of back reaction Wald [3] has developed an axiomatic approach. There one tries to obtain an expression for the renormalized $T_{\mu \nu}$ from the properties (axioms) which it must fulfill. The axioms for the renormalized energy momentum tensor are as follow:

1-For off-diagonal elements standard results should be obtained.

2-In Minkowski space time standard results should be obtained.

3 -Expectation values of energy momentum are conserved.

4-Causality holds .

5-Energy momentum tensor contains no local curvature tensor depending on derivatives of the metric higher than second order.

Two prescriptions that satisfy the first four axioms can differ at most by a conserved local curvature term. Wald [4], showed any prescription for renormalized $T_{\mu \nu}$ which is consistent with axioms 1-4 must yield the given trace up to the addition of the trace of conserved local curvature. It must be noted (that trace anomalies in stress-tensor, that is, the nonvanishing $T_{\mu}^{\mu}$ for a conformally invariant field after renormalization) are originated from some quantum behavior [5]. In two dimensional space time one can show that a trace-free stress tensor can not be consistent with conservation and causality if particle creation occurs [6]. A trace-free, conserved stress tensor in two dimensions must always remain zero if it is initially zero. One can show that it is possible to use an trace anomaly to the energy-momentum tensors of quantum fields under boundary conditions in a black hole background [7], see also [8] as an example which both conformal and gravitational anomalies are present. In this reference the two-dimensional stress tensor have determined by requiring that it is regular at the black hole horizon. It is shown in this paper, that the gravitational anomaly leads to additional flow in the $(r t)$ component of the stress tensor. In four dimensions, just as in two dimensions, a trace-free stress tensor which agrees with the formal expression for the matrix elements between orthogonal states can not be compatible with both conservation laws and causality. Recently, Robinson and Wilczek suggested a new derivation of Hawking radiation from Schwarzschild black holes through gravitational anomalies [9]. The anomaly in field theory occurs if a symmetry of the action or the corresponding conservation law, valid in the classical theory, is violated in the quantized version. A gravitational anomaly is an anomaly in general covariance which shows up as the non-conservation of the energy-momentum tensor. In Robinson-Wilczek work, the Hawking radiation is understood as compensating flux to cancel gravitational anomalies at the horizon $[9,10,11,12,13]$. As have indicated in [10], Robinson-Wilczek approach is effectively equivalent with dilaton-coupled trace anomaly in two dimensions (to see the conformal anomaly for most general $2 D$ dilaton coupled scalar-dilaton system with arbitrary dilaton couplings refer to [14]). 
In 1992 Bañados, Teitelboim and Zanelli (BTZ) [17, 18] showed that $(2+1)$-dimensional gravity has a black hole solution. This black hole is described by two (gravitational) parameters, the mass $M$ and the angular momentum (spin) $J$. It is locally AdS and thus

it differs from Schwarzschild and Kerr solutions since it is asymptotically anti-de-Sitter instead of flat spacetime. Additionally, it has no curvature singularity at the origin. AdS black holes, are members of this two-parametric family of BTZ black holes and they are very interesting in the framework of string theory and black hole physics [19, 20]. The Kaluza-Kelin reduction of $3 D$ gravity with minimal scalars to $2 D$ dilaton-Maxwell gravity with dilaton coupled scalars and the rotating BTZ black hole can be also described by $2 D$ charged dilatonic black hole [21].

In this paper we show that Robinson-Wilczek method can also be applied to a rotating BTZ black holes. We show that the gauge and gravitational anomaly in this black hole background is cancelled by the total flux of a $1+1$ dimensional blackbody at the Hawking temperature of this black hole.

\section{Robinson-Wilczek Approach to the Gravitational Anomalies}

In this section, we will review the gravitational anomaly method $[9,10,11,12,13]$. Consider the metric of the type,

$$
d s^{2}=-f(r) d t^{2}+\frac{1}{f(r)} d r^{2}+r^{2} d \Omega_{D-2}^{2}
$$

where $d^{2} \Omega_{(D-2)}$ is the line element of the (D-2)-dimensional unit sphere and the metric element $f(r)$ depends on the matter distribution. The horizon is located at $r=r_{H}$, where $f\left(r_{H}\right)=0$. The scalar field theory on this metric can be reduced to the 2-dimensional theory. The action of the scalar field is

$$
\begin{aligned}
S[\varphi] & =\frac{1}{2} \int d^{D} x \sqrt{-g} \varphi \nabla^{2} \varphi \\
& =\frac{1}{2} \int d^{D} x r^{D-2} \sqrt{\gamma} \times \varphi\left(-\frac{1}{f} \partial_{t}^{2}+\frac{1}{r^{D-2}} \partial_{r} r^{D-2} f \partial_{r}+\frac{1}{r^{2}} \Delta_{\Omega}\right) \varphi
\end{aligned}
$$

where $\gamma$ is the determinant of $d \Omega_{D-2}^{2}$ and $\Delta_{\Omega}$ is the collection of the angular derivatives. Now we take the limit $r \rightarrow r_{H}$ and leave only dominant terms. Thus, the action becomes

$$
\begin{aligned}
S[\varphi] & =\frac{r_{H}{ }^{D-2}}{2} \int d^{D} x \sqrt{\gamma} \varphi\left(-\frac{1}{f} \partial_{t}^{2}+\partial_{r} f \partial_{r}\right) \varphi \\
& =\sum_{n} \frac{r_{H}{ }^{D-2}}{2} \int d t d r \varphi_{n}\left(-\frac{1}{f} \partial_{t}^{2}+\partial_{r} f \partial_{r}\right) \varphi_{n}
\end{aligned}
$$

in the second line $\varphi$ is expanded by $(D-2)$-dimensional spherical harmonics. This action is infinite set of the scalar fields on the 2-dimensional metric

$$
d s^{2}=-f(r) d t^{2}+\frac{1}{f(r)} d r^{2}
$$


Thus, we can reduce the scalar field theory in $D$-dimensional black hole spacetime to that in 2-dimensional spacetime near the horizon. The gravitational anomaly of the chiral scalar field in $1+1$ dimensions is given as $[15,16]$

$$
\nabla_{\mu} T_{\nu}^{\mu}=\frac{1}{96 \pi \sqrt{-g}} \epsilon^{\beta \delta} \partial_{\delta} \partial_{\alpha} \Gamma_{\nu \beta}^{\alpha}
$$

The aforesaid gravitational anomaly is purely timelike and can be given by

$$
\nabla_{\mu} T_{\nu}^{\mu} \equiv A_{\nu} \equiv \frac{1}{\sqrt{-g}} \partial_{\mu} N_{\nu}^{\mu}
$$

where the quantities $N_{\nu}^{\mu}$ are defined as

$$
N_{\nu}^{\mu}=\frac{1}{96 \pi} \epsilon^{\beta \mu} \partial_{\alpha} \Gamma_{\nu \beta}^{\alpha}
$$

and the epsilon tensor reads

$$
\epsilon^{\mu \nu}=\left(\begin{array}{cc}
0 & 1 \\
-1 & 0
\end{array}\right) .
$$

For the specific Schwarzschild type black hole spacetime described by (1), the components of $N_{\nu}^{\mu}$ are

$$
\begin{aligned}
& N_{t}^{t}=N_{r}^{r}=0 \\
& N_{t}^{r}=\frac{1}{192 \pi}\left(f^{\prime 2}+f^{\prime \prime} f\right) \\
& N_{r}^{t}=-\frac{1}{192 \pi f^{2}}\left(f^{\prime 2}-f^{\prime \prime} f\right) .
\end{aligned}
$$

Therefore the quantity $\Phi$ that describes the pure flux reads

$$
\begin{aligned}
\Phi & =\left.N_{t}^{r}\right|_{r_{H}} \\
& =\frac{1}{192 \pi} f^{\prime 2}\left(r_{H}\right) .
\end{aligned}
$$

It is well known from thermodynamics that the surface gravity $\kappa$ of the specific Schwarzschild type black hole is given by

$$
\begin{aligned}
\kappa & =\left.\frac{1}{2} \frac{\partial f}{\partial r}\right|_{r=r_{H}} \\
& =\frac{1}{2} f^{\prime}\left(r_{H}\right) .
\end{aligned}
$$

It is also known from black hole thermodynamics that the Hawking temperature of this black hole is given as

$$
\begin{aligned}
T_{H} & =\frac{\kappa}{2 \pi} \\
& =\frac{f^{\prime}\left(r_{H}\right)}{4 \pi}
\end{aligned}
$$

while a beam of massless black body radiation moving in the positive radial direction at a temperature $T_{H}$ in a spacetime has a flux of the form

$$
\Phi=\frac{\pi}{12} T^{2}
$$

Therefore it is evident that the flux (11) required to cancel the gravitational anomaly at the horizon is precisely the thermal flux from a black hole at the Hawking temperature. 


\section{Rotating BTZ Black Hole}

The discussion in the previous section can be extended to the rotating BTZ black holes. The black hole solutions of Bañados, Teitelboim and Zanelli $[17,18]$ in $(2+1)$ spacetime dimensions are derived from a three dimensional theory of gravity

$$
S=\int d x^{3} \sqrt{-g}\left({ }^{(3)} R+2 \Lambda\right)
$$

with a negative cosmological constant $\left(\Lambda=\frac{1}{l^{2}}>0\right)$.

The corresponding line element is

$$
d s^{2}=-\left(-M+\frac{r^{2}}{l^{2}}+\frac{J^{2}}{4 r^{2}}\right) d t^{2}+\frac{d r^{2}}{\left(-M+\frac{r^{2}}{l^{2}}+\frac{J^{2}}{4 r^{2}}\right)}+r^{2}\left(d \theta-\frac{J}{2 r^{2}} d t\right)^{2}
$$

whit metric element:

$$
f(r)=\left(-M+\frac{r^{2}}{l^{2}}+\frac{J^{2}}{4 r^{2}}\right)
$$

with $M$ the Arnowitt-Deser-Misner (ADM) mass, $J$ the angular momentum (spin) of the BTZ black hole and $-\infty<t<+\infty, 0 \leq r<+\infty, 0 \leq \theta<2 \pi$.

The outer and inner horizons, i.e. $r_{+}$(henceforth simply black hole horizon) and $r_{-}$ respectively, concerning the positive mass black hole spectrum with spin $(J \neq 0)$ of the line element (18) are given as

$$
r_{ \pm}^{2}=\frac{l^{2}}{2}\left(M \pm \sqrt{M^{2}-\frac{J^{2}}{l^{2}}}\right)
$$

and therefore, in terms of the inner and outer horizons, the black hole mass and the angular momentum are given, respectively, by

$$
M=\frac{r_{+}^{2}}{l^{2}}+\frac{J^{2}}{4 r_{+}^{2}}
$$

and

$$
J=\frac{2 r_{+} r_{-}}{l}
$$

with the corresponding angular velocity to be

$$
\Omega=\frac{J}{2 r^{2}} .
$$

The Hawking temperature $T_{H}$ of the black hole horizon is [22]

$$
\begin{aligned}
T_{H} & =\frac{1}{2 \pi r_{+}} \sqrt{\left(\frac{r_{+}^{2}}{l^{2}}+\frac{J^{2}}{4 r_{+}^{2}}\right)^{2}-\frac{J^{2}}{l^{2}}} \\
& =\frac{1}{2 \pi r_{+}}\left(\frac{r_{+}^{2}}{l^{2}}-\frac{J^{2}}{4 r_{+}^{2}}\right) .
\end{aligned}
$$


If one neglect classically irrelevant ingoing modes near the horizon, the effective twodimensional theory becomes chiral near the horizon and the gauge symmetry or general coordinate covariance becomes anomalous due to the gauge or gravitational anomalies. The current is conserved

$$
\partial_{r} J_{(o)}^{r}=0
$$

outside the horizon. In the region near the horizon, since there are only outgoing fields, the current satisfies the anomalous equation

$$
\partial_{r} J_{H}^{r}=\frac{m^{2}}{4 \pi} \partial_{r} A_{t}
$$

where $m$ is the $U(1)$ charge of two-dimensional massless field. One can solve these equations in each regions as

$$
\begin{gathered}
J_{(o)}^{r}=C_{0} \\
J_{H}^{r}=C_{H}+\frac{m^{2}}{4 \pi}\left(A_{t}(r)-A_{t}\left(r_{+}\right)\right)
\end{gathered}
$$

where $C_{0}$ and $C_{H}$ are integration constants. Under gauge transformations, variation of the effective action is given by

$$
-\delta W=\int d^{2} x \sqrt{-g_{2}} \lambda \nabla_{\mu} J^{\mu}
$$

where $\lambda$ is a gauge parameter, and

$$
J^{\mu}=J_{(o)}^{\mu} \theta_{+}(r)+J_{H}^{\mu} H(r)
$$

here $\theta_{+}(r)=\theta\left(r-r_{+}-\epsilon\right)$ and $H(r)=1-\theta_{+}(r)$. By using the anomaly equation we have

$$
-\delta W=\int d^{2} x \lambda\left[\delta\left(r-r_{+}-\epsilon\right)\left(J_{(0)}^{r}-J_{(H)}^{r}+\frac{m^{2}}{4 \pi} A_{t}\right)+\partial_{r}\left(\frac{m^{2}}{4 \pi} A_{t} H\right)\right] .
$$

The total effective action must be gauge invariant and the last term should be cancelled by quantum effects of the classically irrelevant ingoing modes. The quantum effect to cancel this term induced by the ingoing modes near the horizon. The coefficient of the delta-function should also vanish, which relates the coefficient of the current in two region,

$$
C_{0}=C_{H}-\frac{m^{2}}{4 \pi} A_{t}\left(r_{+}\right)
$$

$C_{H}$ is the value of the consistent current at the horizon. In order to determine the current flow, we need to fix the value of the current at the horizon. Since the covariant current is written as $\tilde{J}^{r}=J^{r}+\frac{m^{2}}{4 \pi} A_{t} H$, the condition $\tilde{J}^{r}\left(r_{+}\right)=0$ determines the value of the charge flux to be

$$
C_{0}=-\frac{m^{2}}{2 \pi} A_{t}\left(r_{+}\right)=-\frac{m^{2}}{2 \pi} \Omega\left(r_{+}\right)=-\frac{m^{2}}{2 \pi} \frac{J}{2 r_{+}^{2}}=-\frac{m^{2} r_{-}}{2 \pi l r_{+}}
$$

Similarly one can determine the flux of the stress tensor radiated from rotating BTZ black holes. The total flux of the energy-momentum tensor is given by

$$
a_{0}=\frac{m^{2}}{4 \pi} A_{t}^{2}\left(r_{+}\right)+N_{t}^{r}\left(r_{+}\right)=\frac{m^{2}}{4 \pi} \Omega^{2}\left(r_{+}\right)+\frac{1}{192 \pi} f^{\prime 2}=\frac{m^{2} r_{-}^{2}}{4 \pi l^{2} r_{+}^{2}}+\frac{1}{192 \pi} f^{\prime 2}
$$


Now we evaluate the quantity $f^{\prime}$ on the horizon. For the rotating BTZ metric this quantity is

$$
\left.f^{\prime}\right|_{r_{+}}=\frac{2 r_{+}}{l^{2}}-\frac{J^{2}}{2 r_{+}^{3}}
$$

By comparing with the Hawking temperature $T_{H}$ of the black hole horizon we see

$$
\left.f^{\prime}\right|_{r_{+}}=4 \pi T_{H}
$$

Then we can rewrite eq.(34) as

$$
a_{0}=\frac{m^{2} r_{-}^{2}}{4 \pi l^{2} r_{+}^{2}}+\frac{\pi}{12} T_{H}^{2}
$$

This value of the flux is the same as the Hawking flux from rotating BTZ black holes.

\section{4 conclusion}

In this paper we have considered a quantum field in a rotating BTZ black hole background. Near the horizon, the field can be effectively described by an infinite collection of $(1+1)$-dimensional fields. In the case of rotating BTZ black hole, the metric is azimuthal symmetric and the angular momentum is conserved. Because of this isometry, the effective two-dimensional theory for each partial wave has $U(1)$ gauge symmetry. The effective background gauge potential for this $U(1)$ symmetry is written in terms of the metric while the quantum field in the rotating BTZ background has a charge $m$ of this gauge symmetry, where $m$ is an angular quantum number. The effective theory is now interpreted as that of charged particles in a charged black hole in $d=2$ [10] (see also [11]). Then we have shown that the Hawking radiation from rotating BTZ blacks holes can be obtain from gauge and gravitational anomalies. We have shown that the gauge

and gravitational anomaly that appears in the BTZ spacetime is cancelled by the total flux of a $1+1$ dimensional blackbody radiation at the Hawking temperature.

\section{References}

[1] J.D. Bekenstein, Lett. Nuovo. Cim. 4, 737 (1972); Phys. Rev. D7, 2333 (1973); Phys. Rev. D9, 3292 (1974).

[2] S.W. Hawking, Comm. Math. Phys. 25, 152 (1972); J.M. Bardeen, B. Carter and S.W. Hawking, Comm. Math. Phys. 31, 161 (1973).

[3] R. M. Wald, Commun. Math. Phys. 54, 1(1977).

[4] R. M. Wald, Phys. Rev.D17, 1477, (1978).

[5] S. Coleman and R. Jackiw, Ann. Phys. (NY)67, 552,(1971).

[6] S. M. Christensen and S. A. Fulling, Phys. Rev. D15, 2088, (1977). 
[7] M. R. Setare, A. H. Rezaeian, Mod. Phys. Lett. A15, 2159, (2000); M. R. Setare, Class. Quant. Grav. 18, 2097, (2001); M. R. Setare Gen. Rel. Grav. 35, 2279, (2003); T. Christodoulakis, G. A. Diamandis, B. C. Georgalas and E. C. Vagenas, Phys. Rev. D 64, 124022 (2001).

[8] S. N. Solodukhin, Phys. Rev. D 74, 024015, (2006).

[9] S. P. Robinson and F. Wilczek, Phys. Rev. Lett. 95, 011303 (2005) gr-qc/0502074.

[10] S. Iso, H. Umetsu and F. Wilczek, Phys. Rev. Lett. 96, 151302 (2006), hepth/0602146.

[11] S. Iso, H. Umetsu and F. Wilczek, hep-th/0606018.

[12] K. Murata, and J. Soda, hep-th/0606069.

[13] E. C. Vagenas, and S. Das, hep-th/0606077.

[14] S. Nojiri, and S. D. Odintsov, Int. J. Mod. Phys. A16, 1015, (2001).

[15] R. A. Bertlmann and E. Kohlprath, Ann. Phys. (N.Y.) 288, 137 (2001).

[16] L. Alvarez-Gaume and E. Witten, Nucl. Phys. B 234, 269 (1984).

[17] M. Bañados, C. Teitelboim and J. Zanelli, Phys. Rev. Lett. 69 (1992) 1849, hepth/9204099.

[18] M. Bañados, M. Henneaux, C. Teitelboim and J. Zanelli, Phys. Rev. D 48 (1993) 1506, gr-qc/9302012.

[19] J. Maldacena, J. Michelson and A. Strominger, JHEP 9902 (1999) 011, hepth/9812073.

[20] M. Spradlin and A. Strominger, JHEP 9911 (1999) 021, hep-th/9904143.

[21] S. Nojiri, and S. D. Odintsov, Mod. Phys. Lett. A13, 2695, (1998); S. Nojiri, and S. D. Odintsov, Phys. Rev. D 59, 044003, (1999).

[22] A. Kumar and K. Ray, Phys. Lett. B 351 (1995) 431, hep-th/9410068. 